\title{
A Biologically Inspired Model for Occluded Patterns
}

\author{
Mohammad Saifullah \\ Department of Computer and Information Science \\ Linkoping University, Sweden \\ Mohammad.saifullaheliu.se
}

\begin{abstract}
In this paper a biologically-inspired model for partly occluded patterns is proposed. The model is based on the hypothesis that in human visual system occluding patterns play a key role in recognition as well as in reconstructing internal representation for a pattern's occluding parts. The proposed model is realized with a bidirectional hierarchical neural network. In this network top-down cues, generated by direct connections from the lower to higher levels of hierarchy, interact with the bottom-up information, generated from the un-occluded parts, to recognize occluded patterns. Moreover, positional cues of the occluded as well as occluding patterns, that are computed separately but in the same network, modulate the top-down and bottom-up processing to reconstruct the occluded patterns. Simulation results support the presented hypothesis as well as effectiveness of the model in providing a solution to recognition of occluded patterns. The behavior of the model is in accordance to the known human behavior on the occluded patterns.
\end{abstract}

Keywords: Vision, Neural network model, Occluded patterns, Biologicallyinspired.

\section{Introduction}

Occlusion is one of the major sources of trouble for pattern recognition systems. When a pattern is occluded by another pattern a recognition system has to face two problems. First, the system receives features from the occluded as well as occluding pattern and needed to distinguish the two. Second, system is deprived of some features belonging to the occluded pattern that might be very crucial for recognition. Performance of a system for recognizing occluded patterns is largely depended on solution to these two problems.

An interesting case of the occlusion arises when occluded part of a pattern has discriminatory role between two or more pattern classes. For example, patterns ' $E$ ' and ' $F$ ' are indistinguishable when lower edge ' ', of ' $E$ ' is occluded. In this paper this occlusion is called 'critical occlusion'. This situation is unsolvable for simple pattern recognition system. When we humans confronts with such a situation we need some additional information (e.g., contextual cues) to predict the true identity of such patterns. Without additional information this problem is unsolvable. Humans utilize different contextual cues to recognize critically as well as simple occluded or ambiguous patterns [1][2]. At the core of this recognition ability lies the architecture of the endowed visual information processing system. 
The visual information processing in humans can be easily understood in terms of two stream hypothesis [3]. These streams are the ventral stream or 'What' pathway and the dorsal stream or 'Where' pathway. The ventral stream is responsible for recognizing and identifying objects while the dorsal deals with the spatial information associated with objects. These two pathways interact with each other to solve different visual tasks.

Now, consider the fig. 1 to recapitulate the most common human behavior on the task of occluding patterns. The first two patterns (from left) are unconcluded and can be easily recognized. The third pattern is an occluded one but we can recognize this pattern and also perceive the occluded parts of the pattern. The fourth pattern has some missing parts. This pattern can be recognized but it is comparatively difficult to perceive the missing parts. The fifth one is a critically-occluded pattern; In this case the exact identity of the pattern cannot be determined. It can be any of the two alphabets ' $\mathrm{O}$ ' or ' $\mathrm{Q}$ '. This type of occlusion requires some additional information (e.g., contextual or semantic cues) to predict the exact identity of the pattern. The last pattern is also critically-occluded but there is a difference comparing to the previous case. The occluding pattern is similar to the potentially occluded feature, i.e., the feature that make alphabet ' $\mathrm{O}$ ' to ' $\mathrm{Q}$ '. Though in this case it is still not possible to decide about the identity of the exact pattern without any extra information, but one feels biased to consider it as a character ' $Q$ '. On the basis of above discussion we hypothesize that: i) Occluding patterns play an important role in recognition of occluded patterns and perception of occluding parts, 2) Occluding patterns helps reconstruct the representation of the occluded pattern in the visual system, which is not the case with pattern having missing parts. On the basis of the above mentioned hypothesis and known architecture of the human visual system a model for the occluded pattern is presented in this paper.

The work in this paper is related to the earlier work [4][5]. Especially, the work in this paper is close to that of Fukushima [6][7], where he used the biologically-inspired hierarchical structure but with only bottom-up processing of information. In that work, the occluding patterns were removed to avoid the unwanted effects of irrelevant features. In this paper, occluding patterns are utilized to recognize and reconstruct the occluding parts in a bidirectional hierarchical neural network.

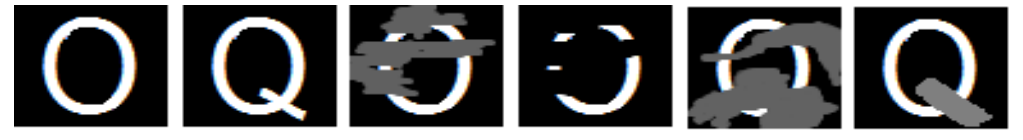

Fig. 1. A few example patterns.

\section{Approach}

Our approach in this work is inspired by the modular and parallel processing architecture of the human brain, where different individual processes go on in parallel having different local goals, but at the same time these processes interact with each other for achieving a common, global goal. More specifically, we model the visual information processing along the two pathways of the human visual system as a 
solution for the recognition of occluding objects. According to this approach the ventral pathway store the representation of the objects along its hierarchical structure. The visible parts of the occluded patterns are processed along the ventral pathway for recognition. This recognition process is facilitated by the top-down cues provided by the direct connections from the lower to higher parts of the hierarchy of the ventral stream. The dorsal pathway encodes the positional information and modulates the ventral pathway to reconstruct the incomplete representation of the occluding parts. This approach requires information to flow not only in bottom-up or top-down direction but all possible direction. In this work this kind of omnidirectional processing is realized by developing a fully recurrent neural network.

\section{The Model}

The model presented in this paper is a modified and extended version of the biologically-inspired models for object recognition [8][9], based on the ventral stream of the human visual system. It has a hierarchical structure of layers of units that interact with each other through feed-forward as well as recurrent connections. The model encodes size, position and shape invariant representation along its hierarchy through learning.

The model proposed in this paper is shown in Fig. 2. The first layer, layer at the lowest level of the hierarchy, is Input layer. The next layer in the model hierarchy is V1. This layer is organized into group of units or hypercolumns such that each group of units looks only at a part of the input or receptive field. This layer sense and encodes the oriented edges in the input images but only from unoccluded parts of the pattern. After V1 the model is divided into two information processing pathways. These two pathways are named after the visual processing pathways of the human brain as the ventral and the dorsal pathways. The ventral pathway process the shape information while the dorsal pathway takes care of the positional information of the input pattern. The ventral pathway is further divided into two information processing channels, i.e., the main channel and the direct channel. The main channel contains the layers V2, V4 and Pat ID while the direct channel is composed of two layers, namely, Direct_Ch and Pat_Category. The V2 layer is also organized into group of units and these groups extract input from the contiguous groups of unit from V1 and thus have somewhat topographic representation of the input pattern. The V4 layer is a single group layer and has receptive field that encompass the whole input image. The last layer in the main channel is the Pat ID layer that interacts with the V4 layer to compute and display the result of processing in this channel. The Direct_Ch get its input from the V1 and project to Pat Category layer. This channel provides a less refined, in terms of discriminatory ability, but fast computing channel to the model for category recognition. The Pat_Category and Pat ID layers are mutually connected to each other. The direct channel computes the object category information that is used as top-down cues to modulate processing along the main channel. This top-down modulation facilitates the processing in the main channel by limiting the output choices for the information processing along the main channel.

The dorsal pathway is composed of only one layer, Pat_Saliency. This layer gets its input from the Input layer and projects to the V2 layer in a topographic manner. 
Ideally, this pathway should get its input from V1 layer but to contain the complexity of the model it is connected to input directly. The Pat_Saliency layer also has a selfconnection to help generate a positional-template of the input pattern. This layer modulates the units of the V2 layer according to the positional-templates of the input images which contains the critically occluded part of the pattern as well. This modulation allows the units in the V2 layer to reconstruct the representation at the occluded locations of the input patterns and hence facilitate the correct recognition of the object identity. Interaction of the Pat_Saliency layer with the V2 layer is based on our hypothesis that how occluding patterns help perceive the occluded pattern in a biologically inspired way.

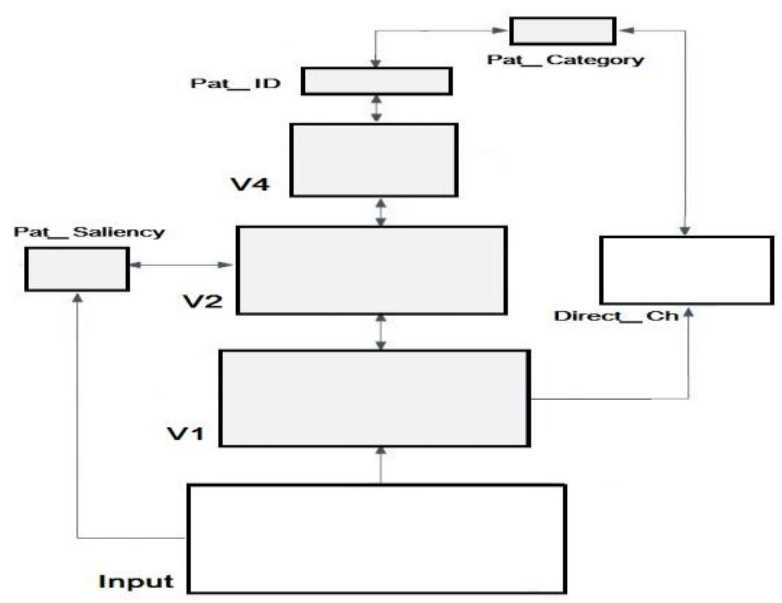

Fig. 2. The proposed biologically-inspired model.

\subsection{Network Algorithm}

An interactive (bidirectional, recurrent) neural network is developed to realize the model. The network was developed in Emergent [10], using the biological plausible algorithm Leabra[8] . Each unit of the network had a sigmoid-like activation function. Learning in the network was based on a combination of Conditional Principal Component Analysis (CPCA), which is a Hebbian learning algorithm and Contrastive Hebbian learning (CHL), which is a biologically-based alternative to back propagation of error, applicable to bidirectional networks [11].

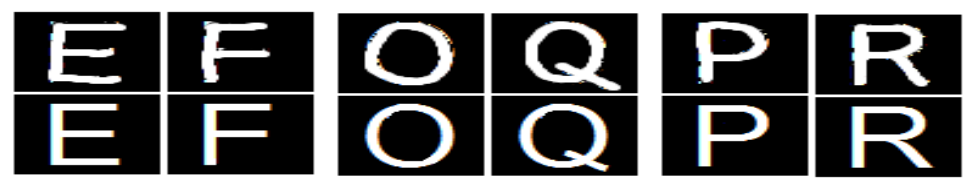

Fig. 3. Patterns used for training. Six different classes of patterns are grouped together into three categories. Close pattern classes in the figure falls in the same category. 


\section{Data for training and testing}

To simplify the training and analysis of the results, gray level images of only six alphabetical character classes (fig. 3) are used for training and testing. These characters are further divided into three categories, such that each category contains two different classes of characters. These categories are created manually on the basis of shape resemblance of classes with each other, so that simulations for criticallyoccluded objects can be easily demonstrated according to the hypothesis presented in this paper.

Tabel 1. Simulation results, when direct channel recognize the category of the object correctly.

\begin{tabular}{|c|c|c|c|}
\hline No. & Pattern Specification & Avg. Correct (\%) & Remarks \\
\hline 1 & No occlusion & 100 & $-\overline{--}$ \\
\hline 2 & Non-critical occlusion & 97 & $\begin{array}{l}\text { A few wrong recognitions when } \\
\text { occlusion is close to critical occlusion }\end{array}$ \\
\hline 3 & Missing Parts & 73 & --- \\
\hline 4 & Critical occlusion & --- & $\begin{array}{l}\text { Arbitrary recognition of a class, } \\
\text { mostly in favor of pattern with } \\
\text { occluded feature }\end{array}$ \\
\hline
\end{tabular}

\section{Simulation and Results}

Training for the two network channels of the ventral pathway, i.e., the main channel and the direct channel, is performed separately. First, the training of the direct channel was performed for learning the three input categories. Second, the main channel was trained on six different classes and three categories. In the second part of the training, the main channel learns representation of each of the six classes as well as a mapping between the Pat_ID and Pat_Category. Each unit of Pat_ID and Pat_Category represent one pattern class and one category respectively in the order they appear in the fig. 3. The dorsal pathway does not require any learning.

After completion of the training, the network is simulated on different occluded patterns, Table 1. In the following section a few simulation results are presented with analysis:

\subsection{Pattern without any occlusion}

Figure 4 shows the behavior of the model when an un-occluded pattern is presented to the network. Since the pattern is complete therefore the main channel can easily recognize it without any additional help from the dorsal pathway or the direct channel. When the pattern is presented to the network, the direct channel activates the correct category of the object (cycle: 6) and modulate the processing in the main ventral pathway, in a top-down manner, by interacting with Pat_ID layer. This action of the direct channel bias limits the choices of the main channel and biases its processing towards class-representations belonging to a specific category (cycle: 1316). Meanwhile, the Pat_Saliency layer of the dorsal pathway encodes the template 
like positional cues of the input pattern and modulates the V2 layer. This result in completing the representation of the pattern first at V2 layer and then in rest of the network (cycle: 5-39). The interaction between the top-down and bottom-up cues as well as with thetemplate like positional cues from the dorsal pathway result in the activation of correct pattern class at the Pat_ID layer (cycle: 17).

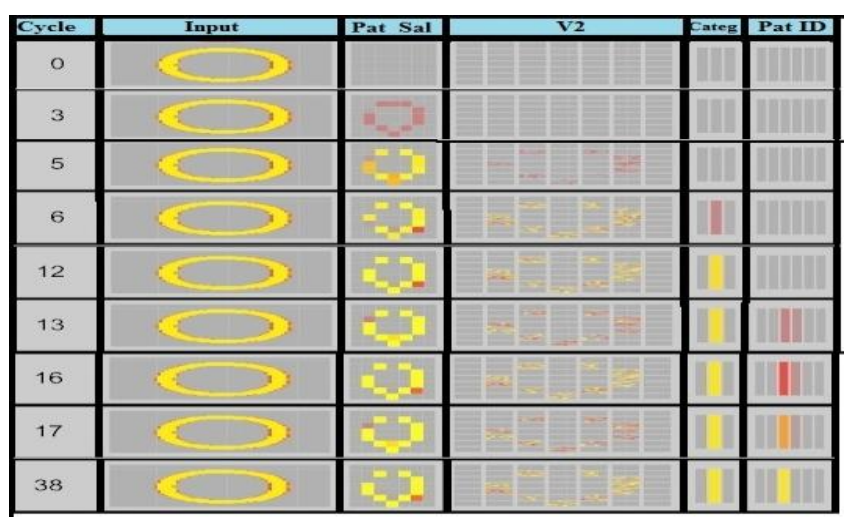

Fig. 4. Snapshots of activations in the various network layers (each layer is made up of a matrix of units, and the activation values of these matrices are shown here). The recorded changes in activation for different processing cycles illustrates how the top-down and bottomup interactions within ventral pathway and interaction between the ventral and the dorsal pathway lead to desired specific behavior. For each graph, in the order from left to right, the columns represent: Number of processing cycle (how far computation of activation has gone), activations in Input layer, Pat_Saliency layer, V2 layer, Pat_Category layer and Pat_ID layer of the network. Yellow (light) colors denote high activation values, red (dark) colors low activation. Gray (neutral) color means no activation.

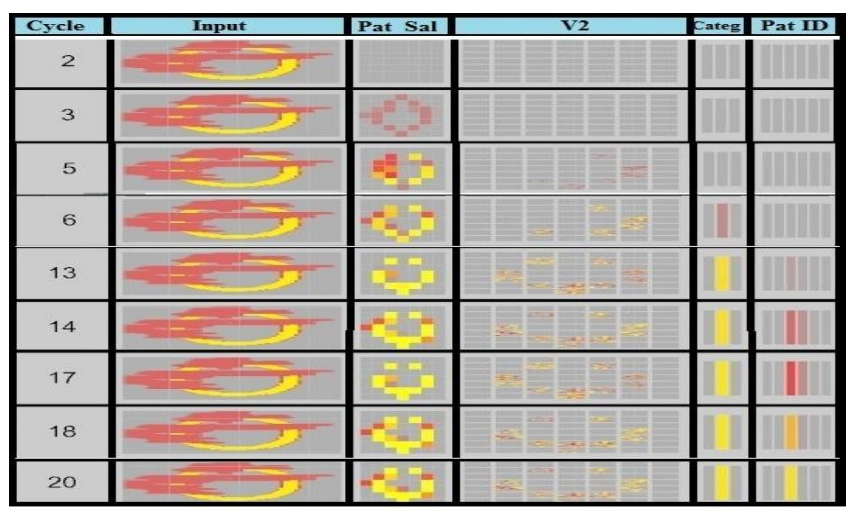

Fig. 5. Cycle-wise activation of various network layers while processing patterns with noncritical occlusion. 


\subsection{Pattern with non-critical occlusion}

In this simulation an image of occluded pattern is presented to the network and the corresponding behavior of the network is shown in the fig. 5. The category of the pattern is correctly recognized at Pat_Category layer (cycle: 13). The role of the dorsal channel is not critical in this case as pattern is not critically-occluded and there is no confusion about the identity of the object. But, Pat_Saliency layer help to reconstruct the representations of the occluding parts of the pattern (cycle: 13-20). Moreover, the result of interaction among different information processing channels in the network lead to correct identification of the pattern class at Pat_ID layer (cycle:14-20).

\subsection{Pattern with missing parts}

Figure 6 shows the behavior of the network when a pattern with missing parts is presented to the network. It is the same pattern that is used in the previous simulation but after removing the occluding patterns. Patterns of activations of Pat ID and Pat_Category layers are similar to the previous case. The main difference of this case from the previous one is the activation patterns at Pat_Saliency and V2 layers. Due to absence of occluding patterns the Pat_Saliency layer does not encode the positional template of the missing parts of the patterns (cycle: 3-51). Consequently, interaction between the Pat_Saliency and the V2 layer does not reconstruct the representation of the missing parts of the occluding pattern (cycle: 5-51). It supports our hypothesis about the role of occluding patterns in reconstructing the occluding parts of the patterns through the dorsal pathway.

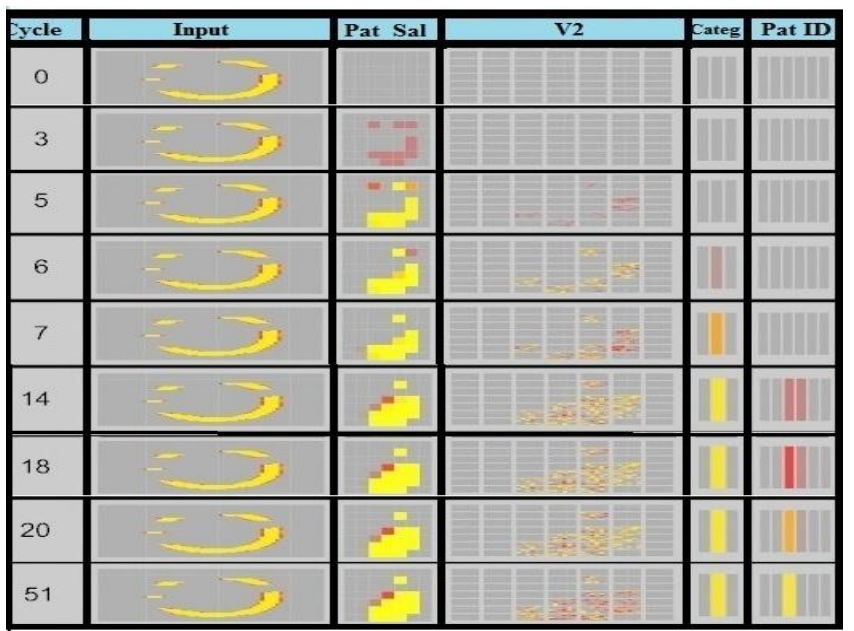

Fig. 6. Cycle-wise activation of various network layers while processing pattern with missing parts 


\subsection{Pattern with critical occlusion}

Figure 7 shows processing of the network for a critically-occluded pattern. Soon after the input is presented to the network the Pat_Category layer categorizes the pattern (cycle: 7) and strengthens the units of the Pat_ID layer that represent the objects of this particular category (cycle: 13-18). The Pat_ID layer in turns modulates the main channel by biasing it for two specific classes of patterns. In this case, the bottom-up cues along the main network belong to the whole category and not to a specific object, as the critical part of the pattern are occluded. In the same way, the top-down cues also belongs to the whole category. In this situation bottom-up and top-down cues will interact along the hierarchy of the main network and activates the representations of the features that are common in both classes. Consequently, the most probable object at the output layer should be the pattern with minimum occluded features, in this case that would be pattern ' $\mathrm{O}$ '. However, the positional cues in the form of pattern template from the dorsal channel make a difference here. Since this template contains the occluded parts of the pattern as well, and modulates the V2 layer units that represent the occluded part of pattern ' $Q$ '. The modulation of critically occluded pattern especially interacts with the top-down category cues and activates the units representing the critically-occluded patterns. The resulting representation in the main network is tilted towards the pattern ' $Q$ ' that is accordingly displayed at the layer Pat_ID. But, as representation of the both classes is present in the network therefore it is just a matter of chance that which pattern will win and accordingly be declared as a final output. It depends on the shape of occluding patterns as well as on the accuracy with which Pat_Saliency layer encodes the positional cues. Sometime, this interaction results in a state where the network cannot decide about the exact class of the pattern and oscillate between the two choices. In such cases some kind of contextual cues are required to bias the result towards a specific class.

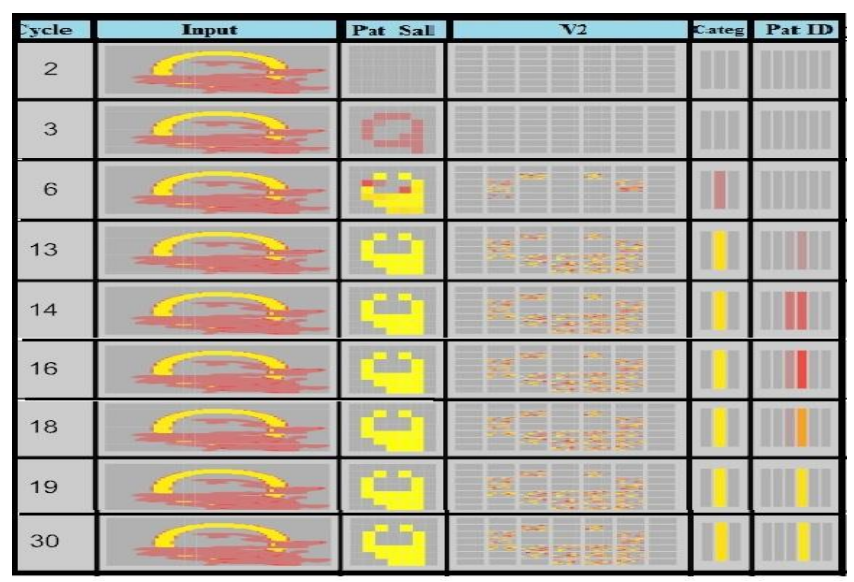

Fig. 7. Cycle-wise activation of various network layers while processing critically occluded pattern 


\section{Conclusion}

In this paper a biologically-inspired model for recognition of occluded pattern is presented. The information processing strategy is based on the hypothesis that occluding patterns provide the important cues for recognition as well as reconstructing the representation of occluded parts. The architecture of the model is based on the two stream hypothesis of human visual system. The computers simulations with the model demonstrate that it provides a satisfactory solution to the occluded patterns recognition as well as produce a behavior that is in accordance to the known human behavior on recognizing partly occluded objects.

\section{$7 \quad$ References}

[1] E. Palmer, "The effects of contextual scenes on the identification of objects," Memory \& Cognition, vol. 3, no. 5, pp. 519-526, 1975.

[2] M. Bar, "Visual objects in context," Nature Reviews Neuroscience, vol. 5, no. 8, pp. 617-629, 2004.

[3] L. G. Ungerleider and J. V. Haxby, "'What" and "where" in the human brain," Current opinion in neurobiology, vol. 4, no. 2, pp. 157-165, 1994.

[4] S. Grossberg and E. Mingolla, "Neural dynamics of perceptual grouping: Textures, boundaries, and emergent segmentations," Attention, Perception, \& Psychophysics, vol. 38, no. 2, pp. 141-171, 1985.

[5] J. S. Lee, C. H. Chen, Y. N. Sun, and G. S. Tseng, "Occluded objects recognition using multiscale features and hopfield neural network," Pattern recognition, vol. 30, no. 1, pp. 113-122, 1997.

[6] K. Fukushima, "Recognition of partly occluded patterns: a neural network model," Biological Cybernetics, vol. 84, no. 4, pp. 251-259, 2001.

[7] K. Fukushima, "Neural network model restoring partly occluded patterns," in Knowledge-Based Intelligent Information and Engineering Systems, pp. 131$138,2003$.

[8] R. C. O'Reilly and Y. Munakata, Computational explorations in cognitive neuroscience: Understanding the mind by simulating the brain. The MIT Press, 2000.

[9] M. Saifullah, "Exploring Biologically-Inspired Interactive Networks for Object Recognition," 2011.

[10] B. Aisa, B. Mingus, and R. O'Reilly, "The emergent neural modeling system," Neural networks, vol. 21, no. 8, pp. 1146-1152, 2008.

[11] R. C. O'Reilly and Y. Munakata, Computational explorations in cognitive neuroscience: Understanding the mind by simulating the brain. The MIT Press, 2000 . 